\title{
Effect of Kidney Function and Dialysis on the Pharmacokinetics and Pharmacodynamics of Roxadustat, an Oral Hypoxia-Inducible Factor Prolyl Hydroxylase Inhibitor
}

\author{
Dorien Groenendaal-van de Meent ${ }^{1}$ (D) $\cdot$ Virginie Kerbusch $^{2} \cdot$ Rudiger Kaspera $^{1} \cdot$ Begona Barroso-Fernandez $^{1}$. \\ Piergiorgio Galletti ${ }^{1} \cdot$ Gernot K. Klein $^{3} \cdot$ Martin den Adel $^{1}$
}

Published online: 9 November 2020

(c) The Author(s) 2020

\begin{abstract}
Background and Objectives Roxadustat is an orally active hypoxia-inducible factor prolyl hydroxylase inhibitor for anemia in chronic kidney disease. The pharmacokinetics, metabolic profile, and pharmacodynamics of roxadustat were investigated in subjects with different degrees of kidney function.

Methods This phase 1 open-label study enrolled subjects with normal and severely impaired kidney function, and end-stage renal disease (ESRD) on continuous ambulatory peritoneal dialysis (CAPD) or automated peritoneal dialysis (APD) or hemodialysis/hemodiafiltration (HD/HDF). All subjects received a single 100-mg dose of oral roxadustat. Within a singlesequence, two-treatment period design (P1/P2), subjects with ESRD on HD/HDF received roxadustat $2 \mathrm{~h}$ after $(\mathrm{P} 1)$ and $2 \mathrm{~h}$ before (P2) a dialysis session. Area under the plasma concentration-time curve (AUC) from administration to infinity $\left(\mathrm{AUC}_{\mathrm{inf}}\right)$, maximum concentration $\left(C_{\mathrm{max}}\right)$, and terminal elimination half-life $\left(t_{1 / 2}\right)$ were assessed for roxadustat; AUC and $C_{\max }$ were assessed for erythropoietin.

Results Thirty-four subjects were enrolled and received roxadustat (normal kidney function, $n=12$; severely impaired kidney function, $n=9$; ESRD on CAPD/APD, $n=1$; ESRD on HD/HDF, $n=12$ ). The geometric least-square mean ratio of $\mathrm{AUC}_{\text {inf }}$ was $223 \%$ and $195 \%$ in subjects with severely impaired kidney function and ESRD on HD/HDF, respectively, relative to subjects with normal kidney function; $C_{\max }$ and $t_{1 / 2}$ were comparable. The pharmacokinetic profile of roxadustat was not affected by HD/HDF. AUC $\mathrm{inf}_{\text {f }}$ and $t_{1 / 2}$ for the metabolites of roxadustat increased in subjects with kidney impairment. The AUC and $C_{\max }$ of erythropoietin increased in subjects with severely impaired kidney function or ESRD on HD/HDF. Roxadustat was well tolerated.

Conclusions Kidney function impairment increased the AUC of roxadustat and its metabolites. The $C_{\max }$ and $t_{1 / 2}$ of roxadustat were comparable among groups. Roxadustat and its metabolites were not cleared by HD/HDF.
\end{abstract}

Clinical Trials Registration Number: NCT02965040.

\section{Introduction}

Electronic supplementary material The online version of this article (https://doi.org/10.1007/s13318-020-00658-w) contains supplementary material, which is available to authorized users.

Dorien Groenendaal-van de Meent

Dorien.Groenendaal@astellas.com

1 Astellas Pharma Europe B.V., Sylviusweg 62, 2333 BE Leiden, The Netherlands

2 PharmAspire B.V., Wijchen, The Netherlands

3 APEX GmbH, Munich, Germany
Anemia is a common complication in patients with chronic kidney disease (CKD). The pathophysiology of CKD anemia has multiple etiologies including the dysregulation of oxygen sensing by the kidney tissue that results in reduced synthesis of erythropoietin, functional iron deficiency, and inflammation. CKD anemia is associated with an increased risk in mortality, morbidity, and hospitalization $[1,2]$. Erythropoiesis-stimulating agents (ESAs) are commonly used for the treatment of anemia in CKD; however, high ESA doses administered to achieve higher hemoglobin $(\mathrm{Hb})$ targets have been associated with increased cardiovascular risk [3]. The safety concerns associated with ESA treatment have resulted in a reduction in their use and dosage worldwide. 


\section{Key Points}

In subjects with severely impaired kidney function or with end-stage renal disease on hemodialysis (HD) or hemodiafiltration (HDF), the mean roxadustat plasma exposure was approximately double compared with subjects with normal renal function, whereas the plasma maximum concentration was not significantly changed; exposure and maximum concentrations of the metabolites of roxadustat increased with kidney function impairment.

Roxadustat and its metabolites were not significantly cleared by HD/HDF in a 4-h dialysis session.

A single 100-mg dose of roxadustat was well tolerated in subjects with different degrees of renal function.

Furthermore, approximately $10 \%$ of CKD patients with anemia do not respond adequately to ESAs and require high ESA doses to achieve target $\mathrm{Hb}$ [4].

Hypoxia-inducible factor prolyl hydroxylase inhibitors (HIF-PHIs) are a class of drugs in clinical development for the treatment of CKD anemia. HIF-PHIs increase Hb levels by activating the body's natural response to hypoxia independent of cellular oxygen levels [5]. Roxadustat is an orally administered HIF-PHI that has shown safety and efficacy in phase 3 trials [6-8], and was recently approved in China for the treatment of anemia in dialysis-dependent and non-dialysis-dependent CKD patients, and in Japan for the treatment of anemia in dialysis-dependent CKD patients. Roxadustat is currently being investigated internationally in both dialysisdependent and non-dialysis-dependent patients.

Roxadustat is a lipophilic acid that is primarily metabolized through phase I oxidation (cytochrome P450 2C8) and phase II conjugation (glucuronidation via uridine diphosphate-glucuronosyltransferases [UGT1A9]). Roxadustat is actively transported into the liver and is excreted by the kidney. Moreover, roxadustat is highly bound (99\%) to proteins in human plasma, mainly albumin [9].

In patients with impaired kidney function, hepatic metabolism and transport may be reduced due to enzyme inhibition by the accumulation of uremic toxins and reduction of the expression of transport proteins and enzymes. The combination of both renal and hepatic dysfunction may contribute to altered pharmacokinetics and clearance of drugs used for CKD treatment [10-12]. Since roxadustat is used in CKD patients with different degrees of kidney function who are non-dialysis-dependent or dialysis-dependent, it is important that the pharmacokinetics of roxadustat and its circulating metabolites are well characterized. This phase 1 study was conducted to investigate the pharmacokinetics, metabolic profile, pharmacodynamics, and safety and tolerability of a single 100-mg dose of roxadustat in subjects with severely impaired kidney function or end-stage renal disease (ESRD), and in subjects with normal kidney function.

\section{Methods}

\subsection{Study Endpoints}

The primary endpoints included evaluating (1) the pharmacokinetics of roxadustat and its circulating metabolites ( $O$-glucuronide-, $O$-glucoside-, and sulphate of hydroxyroxadustat) in the plasma and urine of subjects with normal kidney function, severely impaired kidney function, ESRD on continuous ambulatory peritoneal dialysis (CAPD) or automated peritoneal dialysis (APD), and ESRD on hemodialysis (HD) or hemodiafiltration (HDF); and (2) the effect of dialysis on the pharmacokinetics of roxadustat and its metabolites in subjects with ESRD on HD/HDF. The secondary endpoints were evaluating the pharmacodynamics (assessed by measuring erythropoietin) and safety and tolerability of roxadustat. In addition, a comparison was made for the pharmacokinetics of roxadustat and its circulating metabolites between subjects with severely impaired kidney function or ESRD on HD/HDF and subjects with normal kidney function.

\subsection{Study Design}

This was a phase 1, open-label study conducted at one clinical unit in Germany and one in the United Kingdom between December 2016 and December 2017 (ClinicalTrials.gov Identifier: NCT02965040; EudraCT Number: 2015-00256528). For subjects with normal kidney function, severely impaired kidney function, or those with ESRD on CAPD or APD, the study included a screening period between days -30 and -3 , followed by clinic admission on day -2 , and treatment with a single 100-mg dose of oral roxadustat on day 1. Assessments of the pharmacokinetics of roxadustat and its circulating metabolites ( $O$-glucuronide-, $O$-glucoside-, and sulphate of hydroxy-roxadustat) were conducted in all subjects in plasma (days 1-6) and urine (days 1-4). An end-of-study visit (ESV) occurred 5-9 days after the last assessment or early withdrawal (Fig. 1a). For subjects with ESRD on HD/HDF, a two-treatment period, singlesequence design was used to evaluate the effect of dialysis on the pharmacokinetics of roxadustat. The study included a screening period (day -30 to day -3 ), treatment period 1 (P1, day -2 to day 6$)$, a washout period (1-3 weeks), treatment period 2 (P2, day - 1 to day 6), and an ESV 5-9 days after the last assessment of P2 or early withdrawal. During treatment $\mathrm{P} 1$, subjects were admitted to the clinic on the 
day of the first dialysis session of the week (day - 2) and a single administration of oral roxadustat $100 \mathrm{mg}$ occurred $2 \mathrm{~h}$ after completion of a 4-h dialysis session on day 1 . During treatment $\mathrm{P} 2$, subjects were admitted to the clinic on the day before the second dialysis session of the week (day - 1) and a single administration of oral roxadustat $100 \mathrm{mg}$ occurred $2 \mathrm{~h}$ prior to the start of a 4-hour dialysis session on day 1 . For all patients, a meal was provided approximately $3 \mathrm{~h}$ before administration of roxadustat, after which food and water were not allowed before dosing. After dosing, patients had to refrain from food and water intake for $\geq 2 \mathrm{~h}$. A 100$\mathrm{mg}$ dose of roxadustat was selected because it allowed for potential increases in exposure without any safety concerns based on findings from previous studies [13-15]. In addition, this dose level was considered sufficient for the evaluation of the concentrations of roxadustat and its metabolites. Assessments of the pharmacokinetics of roxadustat and its metabolites were conducted in plasma (days 1-6) and urine (days 1-4) during each treatment period (Fig. 1). Plasma protein binding of roxadustat was assessed on day 1 .

\subsection{Study Population}

Subjects were aged 18-75 years (except for subjects with normal kidney function, aged 40-75 years) with body weight of 45-160 kg. Subjects with normal kidney function had a predose estimated glomerular filtration rate (eGFR) $\geq 90 \mathrm{~mL} /$ $\min / 1.73 \mathrm{~m}^{2}$. Subjects with severely impaired kidney function were not on dialysis and had a predose eGFR $<30 \mathrm{~mL} /$ $\min / 1.73 \mathrm{~m}^{2}$. Subjects with ESRD on CAPD/APD were on CAPD or APD treatment with the same mode of dialysis for $\geq 4$ months prior to admission to the clinic. Subjects with ESRD on HD/HDF were on HD or HDF treatment (three dialysis sessions per week) with the same mode of dialysis for $\geq 4$ months prior to admission to the clinic. Subjects with severely impaired kidney function or ESRD who were previously treated with short-acting ESAs must have discontinued treatment $\geq 14$ days prior to admission.

Subjects with normal kidney function aged $\geq 40$ and $<65$ years were excluded if they had a mean pulse $<45$ or $>90 \mathrm{bpm}$, mean systolic blood pressure (SBP) $<90 \mathrm{mmHg}$ and $>140 \mathrm{mmHg}$, and mean diastolic blood $(\mathrm{DBP})<50 \mathrm{mmHg}$ and $>90 \mathrm{mmHg}$ at day -2 ; whereas, subjects with normal kidney function aged $\geq 65$ and $\leq 75$ years were excluded if they had a mean pulse $<45$ or $>90 \mathrm{bpm}$, mean SBP $<90 \mathrm{mmHg}$ and $>160 \mathrm{mmHg}$, and mean DBP $<50 \mathrm{mmHg}$ and $>100 \mathrm{mmHg}$ at day -2 . Subjects with severely impaired kidney function or ESRD were excluded if they had a mean pulse $<45$ or $>90 \mathrm{bpm}$; mean $\mathrm{SBP}<90 \mathrm{mmHg}$ and $>160 \mathrm{mmHg}$; mean $\mathrm{DBP}<50 \mathrm{mmHg}$

a
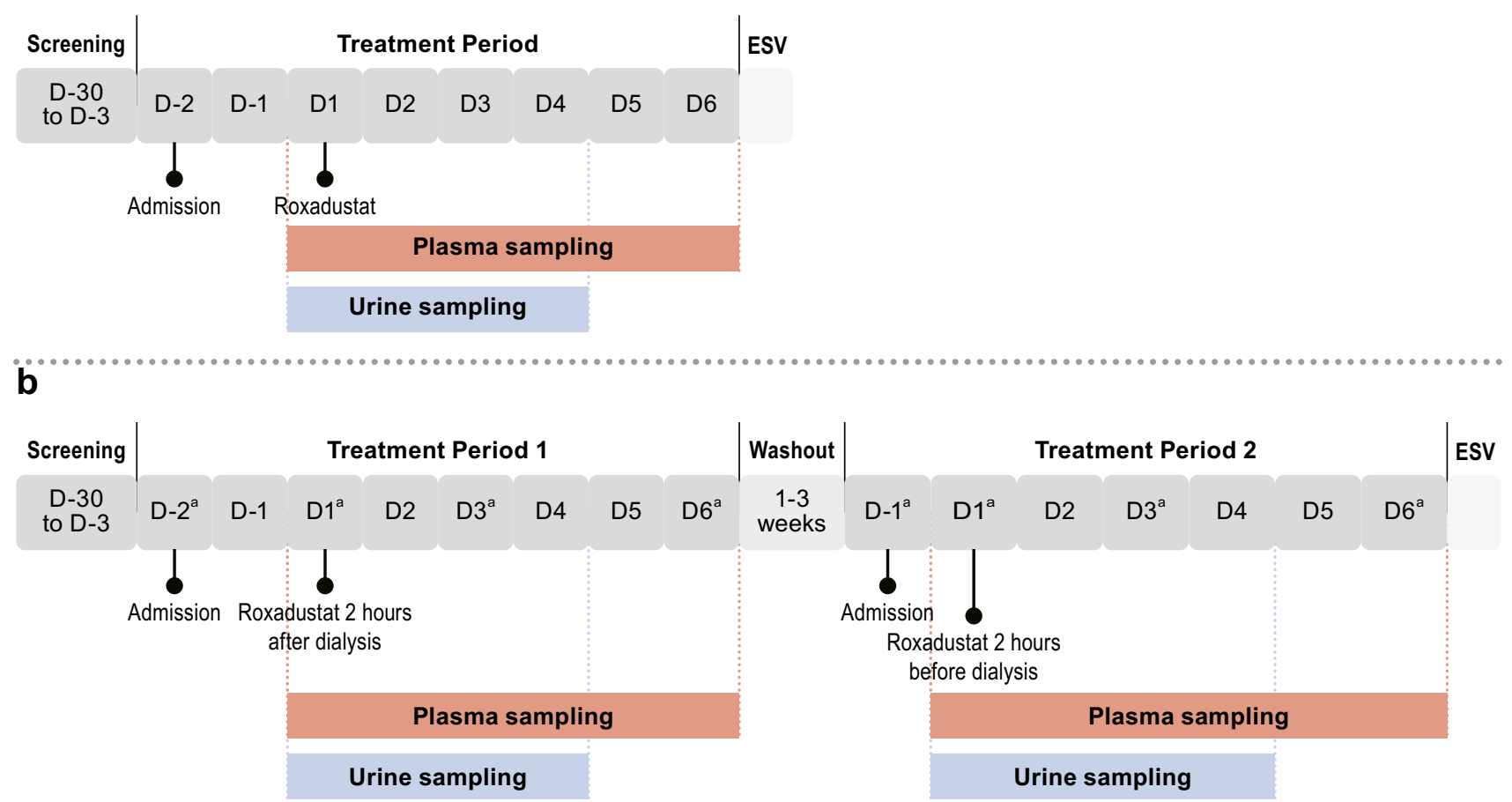

Fig. 1 Study design and schedule of assessments for subjects with normal kidney function, severely impaired kidney function, or subjects with ESRD on CAPD or APD (a) and subjects with ESRD on HD/HDF (b). a Subjects received dialysis treatment. APD automated peritoneal dialysis, $C A P D$ continuous ambulatory peritoneal dialysis, $D$ day, $E S R D$ end-stage renal disease, $E S V$ end-of-study visit, $H D$ hemodialysis, $H D F$ hemodiafiltration 
and $>100 \mathrm{mmHg}$ at day -2 ; if they had used immunosuppressant drugs or drugs to treat malignancies within 3 months prior to admission to the clinical unit; or if they were expected to use oral multivalent cation-containing drugs and mineral supplements, anion-exchange resins, sucralfate or magnesium- or aluminum-containing antacids, phosphate binders and iron-chelating agents, short-acting intravenous or subcutaneous ESA, dapsone, chronic paracetamol $>2 \mathrm{~g}$ per day, or nonsteroidal anti-inflammatory drugs. For all subjects, the use of any nonessential prescribed or nonprescribed drugs within 2 weeks prior to clinic admission was an exclusion criterion.

This study was conducted in accordance with the ethical principles of the Declaration of Helsinki, Good Clinical Practice, the International Council on Harmonisation guidelines, and applicable laws and regulations. The clinical study protocol was reviewed and approved by an Independent Ethics Committee and all subjects signed a written informed consent.

\subsection{Study Assessments}

Serial blood samples were collected to measure the concentrations of roxadustat and its metabolites from predose to $120 \mathrm{~h}$ postdose via a peripherally placed IV cannula or by direct venipuncture in a forearm vein. Blood was drawn in 4-mL tubes containing sodium heparin. For subjects with ESRD on HD/HDF, blood samples were also collected from the arterial and venous access lines from the start to the end of dialysis during P2. Pharmacokinetic assessments for roxadustat in plasma included maximum concentration $\left(C_{\max }\right)$, area under the plasma concentration-time curve from the time of dosing extrapolated to time infinity $\left(\mathrm{AUC}_{\mathrm{inf}}\right)$, apparent total systemic clearance $(\mathrm{CL} / \mathrm{F})$, fraction of parent compound or metabolite available systemically unbound $\left(f_{\mathrm{u}}\right)$, time to $C_{\max }\left(t_{\max }\right)$, terminal elimination half-life $\left(t_{1 / 2}\right)$, effective half-life based on a dosing interval of $48 \mathrm{~h}$ (effective $\left.t_{1 / 2}\right)$, and apparent volume of distribution $\left(V_{\mathrm{z}} / \mathrm{F}\right)$. Pharmacokinetic assessments for the metabolites of roxadustat were $C_{\max }, \mathrm{AUC}_{\mathrm{inf}}, t_{\max }, t_{1 / 2}$, and metabolite to parent ratio (MPR). Urine assessments of roxadustat and its metabolites were conducted only when urine samples were available, and included renal clearance $\left(\mathrm{CL}_{\mathrm{R}}\right)$ and cumulative amount of drug excreted in urine from time of dosing extrapolated to time infinity $\left(\mathrm{Ae}_{\mathrm{inf}}\right)$. Parameters derived from unbound concentrations were calculated in plasma and urine through $f_{\mathrm{u}}$. Urine samples were collected from predose to $72 \mathrm{~h}$ postdose. Dialysate fluid samples were collected from subjects with ESRD on CAPD/APD after each dialysis cycle until $24 \mathrm{~h}$ postdose and from subjects with ESRD on HD/HDF from the start to the end of dialysis. Dialysate samples were used to calculate the dialysis clearance $\left(\mathrm{CL}_{\mathrm{D}}\right)$ for roxadustat and its metabolites, and the fraction of dose cleared by dialysis $\left(f_{\mathrm{D}}\right)$ for roxadustat. Time points for blood, urine, and dialysate sampling are reported in the Supplemental Methods. Concentrations of roxadustat and its metabolites in plasma and urine were measured using liquid chromatography coupled to tandem mass spectrometry with methods previously validated according to the European Medicines Agency Bioanalytical Method Validation Guidance (21 July 2011) [16]. Different analytical methods were used for roxadustat and its metabolites, all of them based on solid phase extraction from the biological matrix followed by separation on a reversed phase $\mathrm{C} 18$ chromatography column and tandem mass spectrometry detection on a 4000 QTrap mass spectrometer using positive turbo ion spray ionization. For each compound, a corresponding stable isotope label was used as internal standard.

Pharmacokinetic parameters were calculated using Phoenix ${ }^{\circledR} 6.2$ (or higher) (Certara US, Inc., Princeton, NJ, USA).

The pharmacodynamics of roxadustat were assessed by measuring the levels of erythropoietin from predose to day 6 of each treatment period (predose to $120 \mathrm{~h}$ postdose). Pharmacodynamic parameters included baseline-corrected maximum observed effect $\left(E_{\max }\right)$, area under the effecttime curve up to the last quantifiable sample corrected for baseline $\left(\mathrm{AUC}_{\mathrm{E} \text {,last }}\right)$, and time of maximum erythropoietin effect corrected for baseline $\left(t_{\mathrm{max}, \mathrm{EPO}}\right)$. Safety was assessed throughout the study by monitoring adverse events (AEs), vital signs, clinical laboratory tests, routine 12-lead electrocardiogram, and 24-h continuous heart rate measurements. An AE was any untoward medical occurrence in a subject administered a study drug, or in those who had undergone study procedures, and which did not necessarily have a causal relationship with this treatment. An AE was considered 'serious' by the investigator if it was life-threatening or if it resulted in death, incapacity to conduct normal life functions, congenital anomaly or birth defect, hospitalization, or other medically important events.

\subsection{Statistical Analysis}

Demographic/baseline characteristics and pharmacokinetic and pharmacodynamic parameters were summarized using descriptive statistics. The pharmacokinetic analysis set (PKAS) included subjects who received at least one dose of roxadustat and had plasma concentrations available to determine $\geq 1$ pharmacokinetic parameter. The pharmacodynamic analysis set (PDAS) included all subjects in the safety analysis set (SAF) who received at least one dose of roxadustat and had plasma concentrations available to determine $\geq 1$ pharmacodynamic parameter. The SAF comprised all enrolled subjects who received $\geq 1$ dose of roxadustat. A linear regression model was used to assess the relationship 
between estimated kidney function (eGFR) and the pharmacokinetics of roxadustat $\left(\mathrm{CL} / \mathrm{F}, \mathrm{CL}_{\mathrm{R}}\right)$ or the pharmacokinetics of its circulating metabolites $\left(\mathrm{CL}_{\mathrm{R}}\right)$. To assess the effect of dialysis on the pharmacokinetics of roxadustat and its metabolites in subjects with ESRD on HD/HDF, the natural logarithmic-transformed $C_{\max }$ and $\mathrm{AUC}_{\text {inf }}$ were analyzed using a mixed-effects analysis of variance (ANOVA) model with treatment period as a fixed effect and subject as a random effect. To assess the effect of kidney function on the pharmacokinetics of roxadustat, the natural logarithmictransformed $C_{\max }$ and $\mathrm{AUC}_{\text {inf }}$ were analyzed using an analysis of covariance (ANCOVA) model with kidney function as a fixed effect and age, sex, and weight as covariates. The targeted population for this study provided adequate precision of the $95 \%$ CI for the regression slope in a simple linear regression modeling of the dependent variable, CL/F, and the independent variable, eGFR. Assuming a standard deviation (SD) of 0.88 and 39.62 for residuals and eGFR, respectively, a sample size of 48 subjects would provide a half-width of 0.0062 for the $95 \%$ CI of the regression slope.

\section{Results}

\subsection{Subject Disposition}

Of 73 screened subjects, 34 (46.6\%) were enrolled in the study and received roxadustat (normal kidney function, $n=12$; severely impaired kidney function, $n=9$; ESRD on CAPD, $n=1$; ESRD on HD/HDF, $n=12$ ). All subjects were included in the SAF, PKAS, and PDAS. Demographics and baseline characteristics are reported in Table 1.

\subsection{Plasma Pharmacokinetics of Roxadustat}

The plasma concentration-time profile for roxadustat showed a slower absorption and initial distribution/elimination phase in subjects with kidney impairment than in those with normal function. In subjects with ESRD on HD/ HDF, there was no meaningful difference in the plasma concentration-time profile of roxadustat between treatment P1 (administration $2 \mathrm{~h}$ after completion of dialysis) and treatment P2 (administration $2 \mathrm{~h}$ before starting dialysis) (Fig. 2). The plasma concentration-time profile of roxadustat for the only subject with ESRD on CAPD was comparable to the one observed for subjects with severely impaired kidney function; no further analysis was conducted for this subject.

$\mathrm{AUC}_{\text {inf }}$ was higher in subjects with severely impaired kidney function and ESRD on HD/HDF compared with those with normal kidney function, whereas mean $C_{\max }$ and $t_{1 / 2}$ were comparable in all groups (Table 2). The mean effective $t_{1 / 2}$ was $9.7 \mathrm{~h}$ in subjects with normal kidney function, $14.9 \mathrm{~h}$ in subjects with severely impaired kidney function, and $15.1 \mathrm{~h}$ and $15.9 \mathrm{~h}$, respectively, in subjects with ESRD on HD/HDF with administration after or before dialysis. The median $t_{\max }$ tended to be shorter in subjects with normal kidney function than in all other groups. $V_{\mathrm{z}} / F$ was similar in subjects with severely impaired kidney function and with ESRD on HD/HDF, and was lower compared with that in subjects with normal kidney function. In subjects with ESRD on HD/HDF, the pharmacokinetic parameters of roxadustat were comparable in treatment $\mathrm{P} 1$ (administration after dialysis) and treatment P2 (administration before dialysis). The mean $f_{\mathrm{u}}$ of roxadustat in plasma was higher in subjects with severely impaired kidney function or with ESRD on HD/ HDF than in those with normal kidney function and was not affected by dialysis (Table 2).

A linear regression analysis of the pharmacokinetic parameters and absolute eGFR of roxadustat showed a positive correlation between $\mathrm{CL} / \mathrm{F}, \mathrm{CL}_{\mathrm{R}}, \mathrm{CL}_{\mathrm{u}} / F$, and $\mathrm{CL}_{\mathrm{R}, \mathrm{u}}$ and eGFR (Supplemental Table S1). An assessment of the effect of kidney function on the pharmacokinetics of roxadustat showed that the geometric least-square mean ratio (GMR) (90\% confidence interval [CI]) of $\mathrm{AUC}_{\text {inf }}$ was $223 \%(185,268)$ in subjects with severely impaired kidney
Table 1 Demographics and baseline characteristics

\begin{tabular}{lccc}
\hline Parameter & $\begin{array}{l}\text { Normal kidney function } \\
(n=12)\end{array}$ & $\begin{array}{l}\text { Severely impaired kidney } \\
\text { function }(n=9)\end{array}$ & $\begin{array}{l}\text { ESRD on HD/ } \\
\text { HDF }(n=12)\end{array}$ \\
\hline Sex, male, $n(\%)$ & $5(41.7)$ & $6(66.7)$ & $9(75.0)$ \\
Race, white, $n(\%)$ & $12(100)$ & $9(100)$ & $12(100)$ \\
Age, years & $60.1(11.4)$ & $64.3(6.6)$ & $56.3(10.8)$ \\
Height, cm & $169.2(9.1)$ & $173.6(9.3)$ & $169.2(7.7)$ \\
Weight, $\mathrm{kg}$ & $77.73(12.40)$ & $90.46(21.08)$ & $76.78(14.58)$ \\
BMI, $\mathrm{kg} / \mathrm{m}^{2}$ & $27.38(5.52)$ & $30.35(8.37)$ & $26.72(4.10)$ \\
eGFR $\left(\mathrm{mL} / \mathrm{min} / 1.73 \mathrm{~m}^{2}\right)$ & $105.74(12.32)$ & $16.91(6.45)$ & $8.59(2.87)$ \\
\hline
\end{tabular}

Data are reported as mean (SD) unless otherwise indicated

$B M I$ body max index, $e G F R$ estimated glomerular filtration rate, ESRD end-stage renal disease, $H D$ hemodialysis, $H D F$ hemodiafiltration, $S D$ standard deviation 

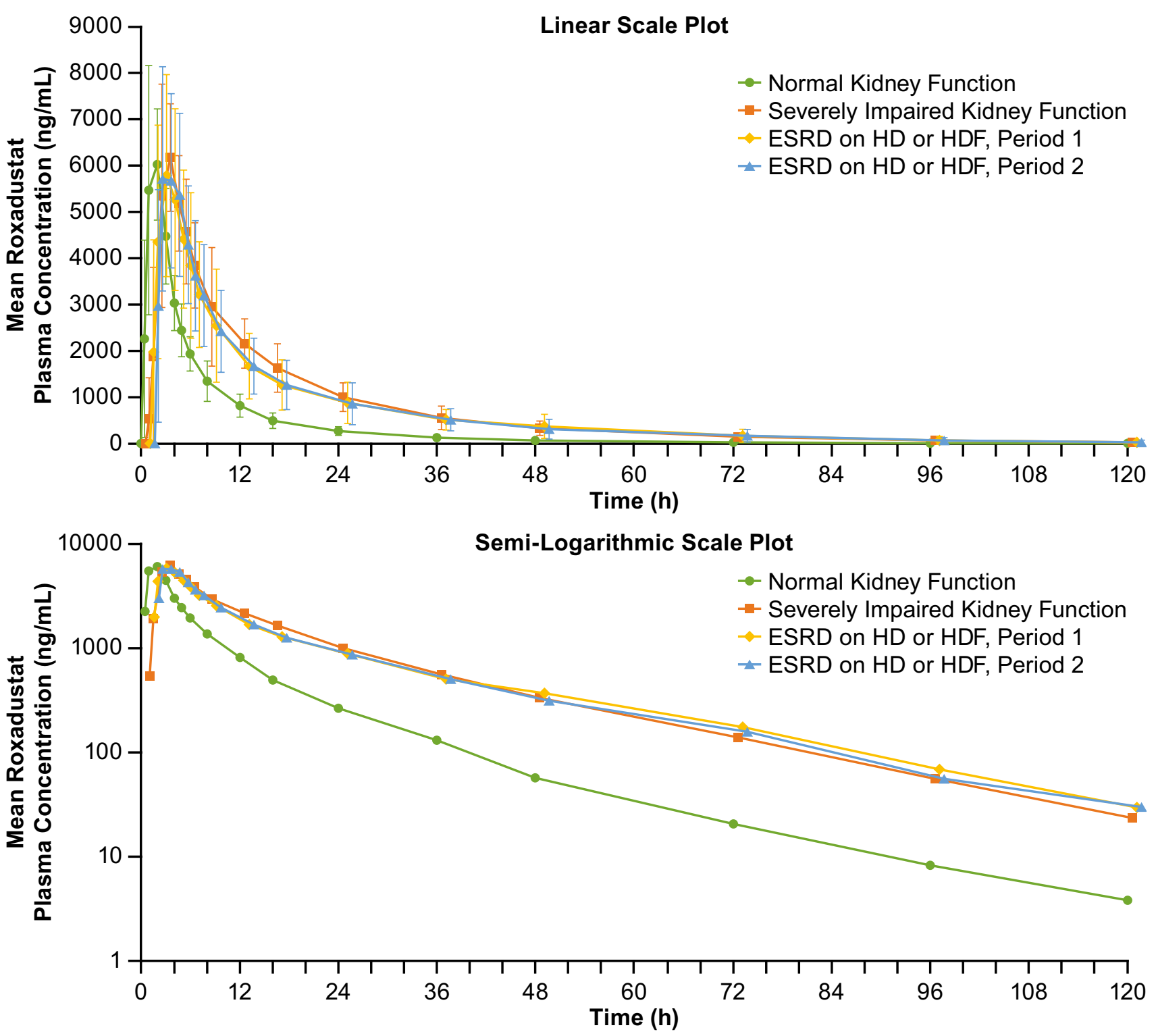

Fig. 2 Mean (SD) plasma concentration-time profiles of roxadustat by renal function group (pharmacokinetic analysis set). ESRD endstage renal disease, $H D$ hemodialysis, $H D F$ hemodiafiltration, $S D$ standard deviation. During P1, subjects received a single roxadustat
(100 mg) administration $2 \mathrm{~h}$ after completion of a dialysis session on day 1 ; during $\mathrm{P} 2$, subjects received a single roxadustat $(100 \mathrm{mg})$ administration $2 \mathrm{~h}$ prior to the start of a dialysis session on day 1

range of $80-125 \%$, suggesting that HD/HDF did not significantly affect the pharmacokinetics of roxadustat (Supplemental Table S3). For subjects with ESRD on HD/HDF (P2), the $\mathrm{CL}_{\mathrm{D}}$ of roxadustat (mean [SD], $0.128[0.0584$ $\mathrm{L} / \mathrm{h}]$ ) was low in comparison with $\mathrm{CL} / \mathrm{F}$. A total of $2.34 \%$ (SD, $1.26 \%$ ) of roxadustat was extracted during dialysis (calculated from the total dose). In addition, the plasma concentrations of roxadustat and metabolites in arterial and venous access lines were comparable (Supplemental Table S4). Thus, neither roxadustat nor its metabolites were significantly removed by a 4-hour dialysis session. 
Table 2 Plasma PK parameters for roxadustat (pharmacokinetic analysis set)

\begin{tabular}{|c|c|c|c|c|}
\hline Parameter & $\begin{array}{l}\text { Normal kidney function } \\
(n=12)\end{array}$ & $\begin{array}{l}\text { Severely impaired kidney } \\
\text { function }(n=9)^{\mathrm{a}}\end{array}$ & $\begin{array}{l}\text { ESRD on HD/HDF treatment } \\
\text { period } 1(n=12)^{\mathrm{b}, \mathrm{c}}\end{array}$ & $\begin{array}{l}\mathrm{ESRD} \text { on } \mathrm{HD} / \mathrm{HDF} \\
\text { treatment period } 2 \\
(n=12)^{\mathrm{b}, \mathrm{c}}\end{array}$ \\
\hline \multicolumn{5}{|l|}{$t_{\max }(\mathrm{h})$} \\
\hline Median & 1.00 & 3.00 & 2.00 & 1.00 \\
\hline Min, $\max$ & $0.983,3.00$ & $1.00,4.00$ & $0.967,5.00$ & $0.967,4.00$ \\
\hline$C_{\max }(\mathrm{ng} / \mathrm{mL})$ & $6780(1,460)$ & $6730(1,310)$ & $6950(1,850)$ & $6910(1,290)$ \\
\hline $\mathrm{AUC}_{\mathrm{inf}}(\mathrm{ng} \cdot \mathrm{h} / \mathrm{mL})$ & $39,800(8,780)$ & $82,500(18,300)$ & $77,400(29,500)$ & $76,600(28,200)$ \\
\hline$t_{1 / 2}(\mathrm{~h})$ & $16.0(6.75)$ & $18.5(4.41)$ & $17.2(6.97)$ & $16.8(5.55)$ \\
\hline $\mathrm{CL} / \mathrm{F}(\mathrm{L} / \mathrm{h})$ & $2.62(0.536)$ & $1.26(0.268)$ & $1.45(0.472)$ & $1.47(0.510)$ \\
\hline$V_{\mathrm{z}} / F(\mathrm{~L})$ & $57.2(15.2)$ & $33.8(10.5)$ & $33.8(11.4)$ & $33.1(8.22)$ \\
\hline$f_{\mathrm{u}}$ & $0.00871(0.000653)$ & $0.0106(0.00121)$ & $0.0110(0.000859)$ & $0.0116(0.00114)$ \\
\hline$C_{\max , \mathrm{u}}(\mathrm{ng} / \mathrm{mL})$ & $59.1(13.4)$ & $70.6(10.3)$ & $76.3(21.1)$ & $80.0(16.4)$ \\
\hline $\mathrm{AUC}_{\mathrm{inf}, \mathrm{u}}(\mathrm{ng} \cdot \mathrm{h} / \mathrm{mL})$ & $348(85.1)$ & $872(203)$ & $850(333)$ & $898(372)$ \\
\hline Effective $t_{1 / 2} 48 \mathrm{~h}(\mathrm{~h})$ & $9.71(1.35)$ & $14.9(2.97)$ & $15.9(4.56)$ & $15.1(4.14)$ \\
\hline
\end{tabular}

Data are expressed as mean (SD) unless otherwise specified

$A U C_{i n f}$ area under the plasma concentration-time curve (AUC) from time of dosing extrapolated to time infinity, $C L / F$ apparent total systemic clearance, $C_{\max }$ maximum concentration, $C_{\max , u} C_{\max }$ for unbound concentration, ESRD end-stage renal disease, $f_{u}$ fraction of parent compound or metabolite available systemically unbound, $H D$ hemodialysis, $H D F$ hemodiafiltration, $P K$ pharmacokinetic, $S D$ standard deviation, $t_{1 / 2}$ terminal elimination half-life, $t_{\max }$ time of maximum concentration, $V / F$ apparent volume of distribution

${ }^{a}$ At $8 \mathrm{~h}$ postdose, one subject had a roxadustat concentration much lower than expected and was excluded

${ }^{\mathrm{b}}$ For one subject, the estimated $f_{\mathrm{u}}$ at $12 \mathrm{~h}$ postdose in treatment period 2 deviated $>5$-fold from the values in treatment period 1 ; therefore, only values from treatment period 1 were used to calculate unbound parameters

${ }^{\mathrm{c}}$ During treatment period 1 , subjects received a single roxadustat $(100 \mathrm{mg})$ administration $2 \mathrm{~h}$ after completion of a dialysis session on day 1 ; during treatment period 2 , subjects received a single roxadustat $(100 \mathrm{mg})$ administration $2 \mathrm{~h}$ prior to the start of a dialysis session on day 1

\subsection{Plasma Pharmacokinetics of the Metabolites of Roxadustat}

The plasma concentration-time profiles of the three circulating metabolites of roxadustat are represented in Fig. 3. In all groups, the plasma concentrations of all three metabolites were low compared with their parent, roxadustat, with mean MPR $<1 \%$ for $O$-glucuronide- and $O$-glucoside-roxadustat, and $<10 \%$ for sulphate of hydroxy-roxadustat (Table 3 ). The mean $\mathrm{AUC}_{\text {inf }}$ for all three metabolites was higher in subjects with severely impaired kidney function and with ESRD on $\mathrm{HD} / \mathrm{HDF}$ (P1 and P2) than in those with normal kidney function. The mean $C_{\max }$ of $O$-glucoside-roxadustat was higher in subjects with severely impaired kidney function and with ESRD on HD/HDF, whereas the lowest mean $C_{\text {max }}$ for sulphate of hydroxy-roxadustat was observed in subjects with severely impaired kidney function. For all three metabolites, the highest mean $t_{1 / 2}$ was observed in subjects with severely impaired kidney function and with ESRD on HD/HDF.

A linear regression analysis showed a positive correlation between $\mathrm{CL}_{\mathrm{R}}$ and eGFR for all three of the metabolites of roxadustat (Supplemental Table S1). Relative to subjects with normal kidney function, the GMRs for $\mathrm{AUC}_{\mathrm{inf}}$ for $O$-glucuronide-, $O$-glucoside-, and sulphate of hydroxy-roxadustat were $174 \%, 376 \%$, and $210 \%$, respectively, in subjects with severely impaired kidney function; and 253\%, 294\%, and $227 \%$, respectively, in subjects with ESRD on HD/HDF. The same ratios for $C_{\max }$ for $O$-glucuronide-, $O$-glucoside-, and sulphate of hydroxy-roxadustat were $74 \%, 170 \%$, and $87 \%$, respectively, in subjects with severely impaired kidney function; and $101 \%, 132 \%$, and $107 \%$, respectively, in those with ESRD on HD/HDF (Supplemental Table S2). The GMRs (P1/P2) for $\mathrm{AUC}_{\text {inf }}$ for $O$-glucuronide-, $O$-glucoside-, and sulphate of hydroxy-roxadustat were $89 \%, 90 \%$, and $96 \%$, respectively; the same ratios for $C_{\max }$ were $98 \%, 101 \%$, and $102 \%$, respectively. For all metabolites, both $\mathrm{AUC}_{\mathrm{inf}}$ and $C_{\max }$ were within the equivalence range of $80-125 \%$, suggesting that HD or HDF did not significantly affect the exposure of the circulating metabolites of roxadustat (Supplemental Table S3).

\subsection{Urine Pharmacokinetics}

Mean $\mathrm{Ae}_{\text {inf }}$ and $\mathrm{CL}_{\mathrm{R}}$ of roxadustat were low in subjects with normal kidney function and decreased in subjects with severe kidney impairment or with ESRD on HD/HDF. $O$-glucuronide-roxadustat was the predominant metabolite in the urine of subjects with normal kidney function. For all metabolites, $\mathrm{Ae}_{\mathrm{inf}}$ and $\mathrm{CL}_{\mathrm{R}}$ decreased with deteriorating kidney function (Table 4). 

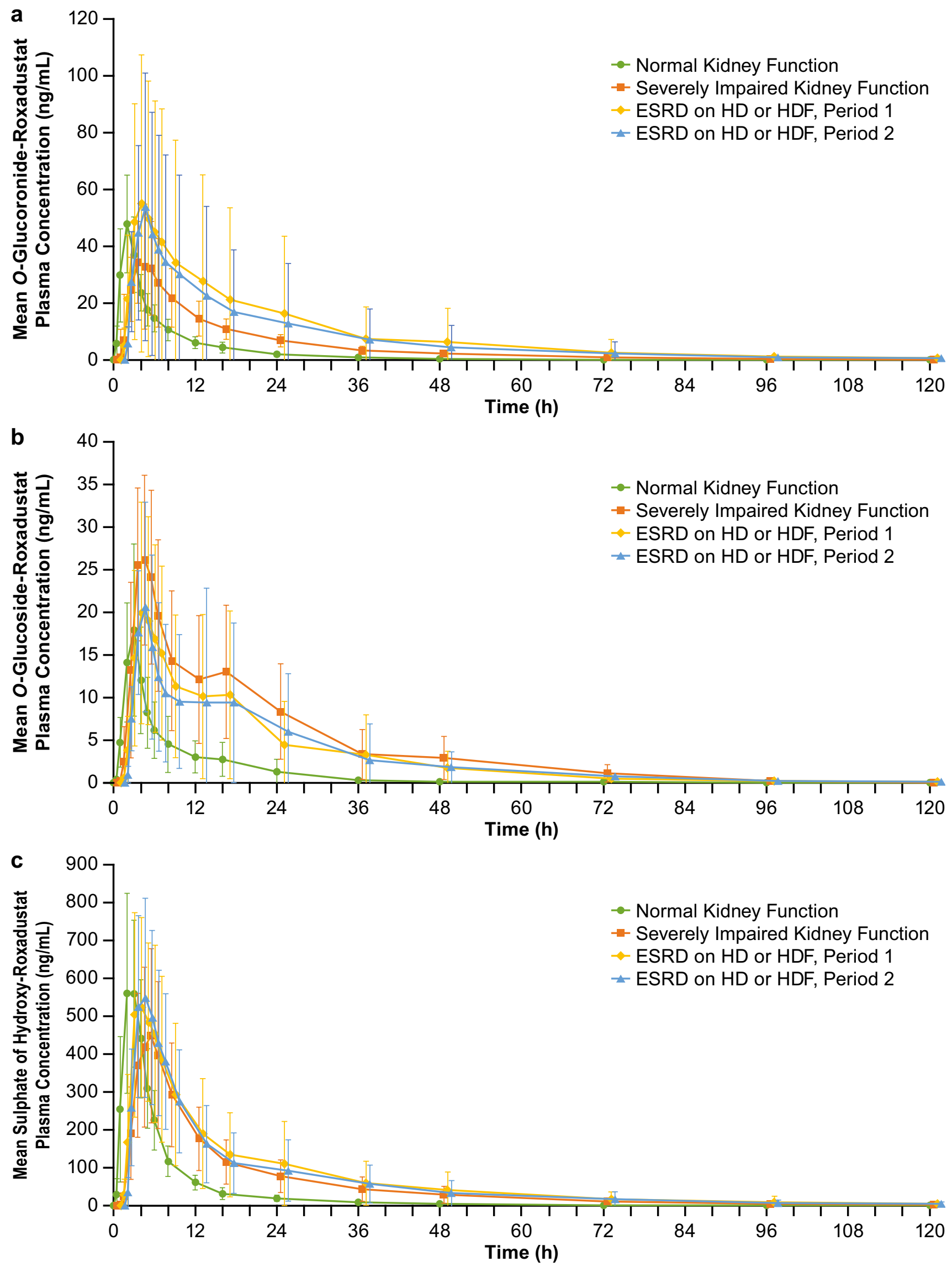
४Fig. 3 Mean (SD) plasma concentration-time profiles of $O$-glucuronide-roxadustat (a), $O$-glucoside-roxadustat (b), and sulphate of hydroxy-roxadustat (c) (pharmacokinetic analysis set). ESRD endstage renal disease, $H D$ hemodialysis, $H D F$ hemodiafiltration; $S D$ standard deviation. During treatment period 1, subjects received a single roxadustat $(100 \mathrm{mg})$ administration $2 \mathrm{~h}$ after completion of a dialysis session on day 1 ; during treatment period 2 , subjects received a single roxadustat $(100 \mathrm{mg})$ administration $2 \mathrm{~h}$ prior to the start of a dialysis session on day 1

\subsection{Plasma Pharmacodynamics of Erythropoietin}

The median baseline-corrected $\mathrm{AUC}_{\mathrm{E}, \text { last }}$ and $\mathrm{E}_{\max }$ for erythropoietin were higher in subjects with severely impaired kidney function or in those with ESRD on HD/HDF than in subjects with normal kidney function. The median $t_{\max }$ for erythropoietin did not differ between kidney function groups and ranged between 8 and $12 \mathrm{~h}$. The levels of erythropoietin returned to near baseline at $24 \mathrm{~h}$ postdose in subjects with normal kidney function, but remained above baseline until $36-48 \mathrm{~h}$ postdose in subjects with kidney impairment. Furthermore, when roxadustat was administered before dialysis (P2), the erythropoietin mean baseline-corrected $\mathrm{AUC}_{\mathrm{E} \text {,last }}$ and $E_{\max }$ were higher than those observed when roxadustat was administered after dialysis (P1) (Table 5).

\subsection{Safety}

No deaths or treatment-emergent AEs (TEAEs) leading to study withdrawal were reported throughout the study. A serious TEAE of anemia was reported by a 59-year-old white female with severely impaired kidney function. The event occurred after a urinary tract infection, with signs of progressive anemia observed following the screening period. Anemia required transfusion with erythrocyte concentrates and was considered resolved after the study completion. Across all kidney function groups, TEAEs reported by $\geq 1$ subject were headache, diarrhea, nausea, asthenia, and urinary tract infection. Continuous heart rate monitoring did not show clinically relevant changes in mean heart rate at time-matched points on day 1 compared with baseline (day - 1).

\section{Discussion}

Roxadustat is an effective treatment for CKD anemia that is prescribed for CKD patients with different degrees of kidney function. This study evaluated whether the pharmacokinetics, metabolic profile, and pharmacodynamics of a single 100-mg dose of oral roxadustat are affected by the degree of kidney dysfunction/impairment, and investigated the effect of dialysis on the pharmacokinetics of roxadustat and its metabolites in subjects with ESRD on CAPD/APD or HD/HDF. Only one subject with ESRD on CAPD was enrolled in this study, therefore it was not possible to draw any statistical conclusions regarding this group. However, the pharmacokinetics of this subject tended to be similar to the pharmacokinetics of subjects with severely impaired kidney function. The exposure to roxadustat was approximately double in subjects with severely impaired kidney function or with ESRD on HD/HDF compared with those with normal kidney function, whereas $C_{\max }$ was not affected. This finding suggests that CKD affects nonrenal elimination of roxadustat, possibly due to the presence of elevated uremic toxins in circulation and inflammation that lead to altered expression and/or activities of plasma proteins, drug-metabolizing enzymes, and drug transporters $[17,18]$. Roxadustat was mainly excreted as metabolites and the excretion of roxadustat itself was low. The elimination rate of roxadustat did not change with kidney function; however, the shape of the mean plasma concentration-time profiles was different in patients with impaired kidney function, with a slower absorption and initial distribution/elimination phase compared with subjects with normal kidney function. This is reflected in the increased effective $t_{1 / 2}$ in subjects with severely impaired kidney function or with ESRD on HD/HDF compared with those with normal kidney function. For drugs with multicompartmental kinetics such as roxadustat, the effective $t_{1 / 2}$ is considered a more appropriate parameter than $t_{1 / 2}$ to predict drug accumulation [19]. Since the effective half-life considers the entire plasma concentration-time profile of the drug, it may better reflect total clearance. The fraction of unbound roxadustat in plasma was higher in subjects with severely impaired kidney function or with ESRD on HD/HDF than in those with normal kidney function. The $\mathrm{AUC}_{\mathrm{inf}}$ and terminal $t_{1 / 2}$ of the three circulating metabolites were higher in subjects with severely impaired kidney function or with ESRD on HD/HDF than in subjects with normal kidney function. For all metabolites of roxadustat, the mean MPR was comparable in all kidney function groups and was $<1 \%$ for $O$-glucuronideand $O$-glucoside-roxadustat, and $<10 \%$ for sulphate of hydroxy-roxadustat, indicating the low levels of all three metabolites. The $\mathrm{CL}_{\mathrm{R}}$ and the amount of roxadustat and its metabolites excreted in urine were lower in subjects with severely impaired kidney function or with ESRD on HD/HDF than in those with normal kidney function. Furthermore, the $\mathrm{CL}_{\mathrm{R}}$ of roxadustat and its metabolites decreased with decreasing eGFR. The exposure of roxadustat was not affected by HD/HDF, as would be predicted from the high plasma protein binding of roxadustat, and the metabolites of roxadustat were not significantly cleared by HD/HDF. In line with an increased roxadustat exposure, the baseline-corrected median erythropoietin AUC E,last was higher in subjects with severely impaired kidney 
Table 3 Plasma PK parameters for metabolites of roxadustat (pharmacokinetic analysis set)

\begin{tabular}{|c|c|c|c|c|}
\hline Parameter & $\begin{array}{l}\text { Normal kidney function } \\
(n=12)\end{array}$ & $\begin{array}{l}\text { Severely impaired kidney } \\
\text { function }(n=9)\end{array}$ & $\begin{array}{l}\text { ESRD on HD/HDF treatment } \\
\text { period } 1^{\text {a }}(n=12)\end{array}$ & $\begin{array}{l}\text { ESRD on } \mathrm{HD} / \mathrm{HDF} \\
\text { treatment period } 2^{\mathrm{b}} \\
(n=12)\end{array}$ \\
\hline \multicolumn{5}{|l|}{$O$-glucuronide-roxadustat } \\
\hline \multicolumn{5}{|l|}{$t_{\max }(\mathrm{h})$} \\
\hline Median (min, $\max )$ & $2.00(1.00,3.00)$ & $3.00(3.00,5.00)$ & $2.00(1.00-8.00)$ & $2.01(1.95,5.00)$ \\
\hline$C_{\max }(\mathrm{ng} / \mathrm{mL})$ & $49.9(16.0)$ & $38.1(19.9)$ & $59.2(50.9)$ & $55.1(43.9)$ \\
\hline $\mathrm{AUC}_{\mathrm{inf}}(\mathrm{ng} \cdot \mathrm{h} / \mathrm{mL})$ & $286(88.2)$ & $533(320)$ & $1,080(1,490)$ & $924(1,240)$ \\
\hline$t_{1 / 2}(\mathrm{~h})$ & $8.10(1.86)$ & $15.9(5.35)$ & $17.5(9.19)$ & $17.2(5.97)$ \\
\hline MPR & $0.00495(0.00159)$ & $0.00432(0.00224)$ & $0.00779(0.00743)$ & $0.00666(0.00607)$ \\
\hline \multicolumn{5}{|l|}{$O$-glucoside-roxadustat } \\
\hline \multicolumn{5}{|l|}{$t_{\max }(\mathrm{h})$} \\
\hline Median (min, max) & $2.99(1.97,3.00)$ & $3.00(3.00,4.00)$ & $3.00(2.00,8.00)$ & $3.00(1.95,12.0)$ \\
\hline$C_{\max }(\mathrm{ng} / \mathrm{mL})$ & $18.3(9.85)$ & $27.6(9.98)$ & $21.7(12.4)$ & $21.9(11.7)$ \\
\hline $\mathrm{AUC}_{\text {inf }}(\mathrm{ng} \cdot \mathrm{h} / \mathrm{mL})$ & $146(87.8)^{\mathrm{c}}$ & $495(296)$ & $369(346)$ & $375(391)^{d}$ \\
\hline$t_{1 / 2}(\mathrm{~h})$ & $8.48(2.97)^{\mathrm{c}}$ & $15.2(3.56)$ & $14.6(5.99)$ & $15.4(4.36)^{\mathrm{d}}$ \\
\hline MPR & $0.00246(0.00126)^{\mathrm{c}}$ & $0.00406(0.00195)$ & $0.00297(0.00159)$ & $0.00296(0.00193)^{\mathrm{d}}$ \\
\hline \multicolumn{5}{|c|}{ Sulphate of hydroxy-roxadustat } \\
\hline \multicolumn{5}{|l|}{$t_{\max }(\mathrm{h})$} \\
\hline Median (min, max) & $2.02(1.97,4.00)$ & $5.00(3.00,6.00)$ & $2.00(1.97,12.0)$ & $2.00(1.95,5.98)$ \\
\hline$C_{\max }(\mathrm{ng} / \mathrm{mL})$ & $635(230)$ & $453(225)$ & $623(222)$ & $627(193)$ \\
\hline $\mathrm{AUC}_{\mathrm{inf}}(\mathrm{ng} \cdot \mathrm{h} / \mathrm{mL})$ & $3,590(1,150)$ & $6,390(2,840)$ & $8,310(5,910)$ & $7,720(4,690)$ \\
\hline$t_{1 / 2}(\mathrm{~h})$ & $15.9(6.12)$ & $18.6(6.72)$ & $18.2(6.59)$ & $18.7(5.74)$ \\
\hline MPR & $0.0725(0.0227)$ & $0.0606(0.0227)$ & $0.0779(0.0256)$ & $0.0747(0.0208)$ \\
\hline
\end{tabular}

Data are expressed as mean (SD) unless otherwise specified

$A U C_{i n f}$ area under the plasma concentration-time curve from the time of dosing extrapolated to time infinity, $C_{\max }$ maximum concentration, $E S R D$ end-stage renal disease, $H D$, hemodialysis, $H D F$ hemodiafiltration, $M P R$ metabolite to parent ratio; $P K$, pharmacokinetic, $S D$ standard deviation, $t_{1 / 2}$ terminal elimination half-life, $t_{\max }$ time of maximum concentration

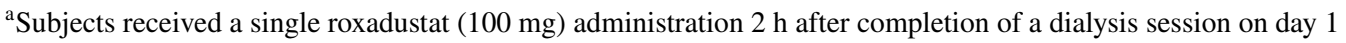

${ }^{\mathrm{b}}$ Subjects received a single roxadustat $(100 \mathrm{mg}$ ) administration $2 \mathrm{~h}$ prior to the start of a dialysis session on day 1

${ }^{\mathrm{c}} n=10$

${ }^{\mathrm{d}} n=11$

function than in those with normal kidney function, and erythropoietin levels remained above baseline longer. This increase was expected since, by inhibiting the enzyme HIF prolyl hydroxylase, roxadustat is able to stabilize the oxygen-sensitive transcription factor HIF- $\alpha$. In the presence of normal oxygen levels, HIF- $\alpha$ is degraded by the activity of the HIF prolyl hydroxylase enzyme, whereas during hypoxia, this enzyme is inhibited, allowing HIF- $\alpha$ to dimerize with HIF- $\beta$, which results in increased expression of erythropoietin, as well as increased iron absorption and mobilization. Therefore, roxadustat is able to stimulate an erythropoietic response by activating the body's natural response to hypoxia in the presence of normal cellular oxygen levels. Higher exposure of roxadustat in subjects with severely impaired kidney function versus those with normal kidney function is therefore expected to increase erythropoietin expression [20].
A single 100-mg dose of roxadustat was well tolerated in subjects with different degrees of kidney function. A serious TEAE of anemia occurred after a urinary tract infection in a patient with severely impaired kidney function. Possible limitations of this study include the small number of subjects (all white) enrolled in each group and the lack of statistical information on subjects with ESRD on CAPD or APD due to the enrollment of only one subject.

With this study we have obtained insight into the pharmacokinetics and pharmacodynamics of roxadustat after administration to patients. Furthermore, the finding that roxadustat and its metabolites were not cleared by HD/HDF suggests that roxadustat may be effectively administered either before or after dialysis. A clinical implication of this study is that roxadustat was well tolerated in all subjects, regardless of the degree of kidney impairment. 
Table 4 Urine pharmacokinetic parameters of roxadustat and its metabolites by renal function group (pharmacokinetic analysis set)

\begin{tabular}{|c|c|c|c|c|}
\hline Parameter & Normal kidney function & $\begin{array}{l}\text { Severely impaired } \\
\text { kidney function }\end{array}$ & $\begin{array}{l}\mathrm{ESRD} \text { on } \mathrm{HD} / \mathrm{HDF} \\
\text { treatment period } 1^{\mathrm{a}}\end{array}$ & $\begin{array}{l}\mathrm{ESRD} \text { on } \mathrm{HD} / \mathrm{HDF} \\
\text { treatment period } 2^{\mathrm{b}}\end{array}$ \\
\hline \multicolumn{5}{|l|}{ Roxadustat } \\
\hline$n$ & 12 & 9 & 8 & 8 \\
\hline $\mathrm{CL}_{\mathrm{R}}(\mathrm{L} / \mathrm{h})$ & $0.0260(0.0159)$ & $0.00949(0.00648)$ & $0.00138(0.00131)$ & $0.00118(0.00112)$ \\
\hline $\mathrm{Ae}_{\mathrm{inf}}(\%)$ & $0.978(0.531)$ & $0.773(0.588)$ & $0.1000(0.101)$ & $0.104(0.145)$ \\
\hline \multicolumn{5}{|c|}{$O$-glucuronide-roxadustat } \\
\hline$n$ & 12 & 9 & 8 & 8 \\
\hline $\mathrm{CL}_{\mathrm{R}}(\mathrm{L} / \mathrm{h})$ & $114(44.3)$ & $30.5(21.0)$ & $2.58(2.96)$ & $2.12(2.57)$ \\
\hline $\mathrm{Ae}_{\text {inf }}(\%)$ & $20.3(5.69)$ & $8.52(4.46)$ & $0.861(0.849)$ & $0.677(0.703)$ \\
\hline \multicolumn{5}{|c|}{$O$-glucoside-roxadustat } \\
\hline$n$ & 10 & 9 & 7 & 7 \\
\hline $\mathrm{CL}_{\mathrm{R}}(\mathrm{L} / \mathrm{h})$ & $99.8(49.2)$ & $13.3(13.8)$ & $1.77(1.61)$ & $1.86(2.59)$ \\
\hline $\mathrm{Ae}_{\text {inf }}(\%)$ & $7.21(2.25)^{\mathrm{c}}$ & $2.83(1.31)$ & $0.281(0.217)$ & $0.270(\mathrm{NA})^{\mathrm{d}}$ \\
\hline \multicolumn{5}{|c|}{ Sulphate of hydroxy-roxadustat } \\
\hline$n$ & 12 & 9 & 7 & 7 \\
\hline $\mathrm{CL}_{\mathrm{R}}(\mathrm{L} / \mathrm{h})$ & $0.735(0.210)$ & $0.0852(0.0452)$ & $0.0121(0.00685)$ & $0.00895(0.00865)$ \\
\hline $\mathrm{Ae}_{\text {inf }}(\%)$ & $2.00(0.746)$ & $0.397(0.229)$ & $0.0594(0.0414)$ & $0.0382(0.0429)$ \\
\hline
\end{tabular}

Data are expressed as mean (SD) unless otherwise specified

$A e_{i n f}$ cumulative amount of drug excreted in urine from time of dosing extrapolated to time infinity, $C L_{R}$ renal clearance, $E S R D$ end-stage renal disease, $H D$ hemodialysis, $H D F$ hemodiafiltration, $S D$ standard deviation

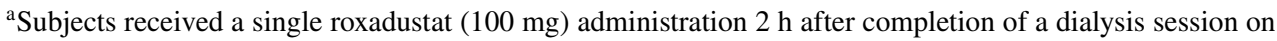
day 1

${ }^{\mathrm{b}}$ Subjects received a single roxadustat $(100 \mathrm{mg})$ administration $2 \mathrm{~h}$ prior to the start of a dialysis session on day 1

${ }^{\mathrm{c}} n=10$

${ }^{\mathrm{d}} n=6$; percentage was not calculated due to missing values for $50 \%$ of patients

Table 5 Plasma pharmacodynamic parameters of erythropoietin (pharmacodynamic analysis set)

\begin{tabular}{|c|c|c|c|c|}
\hline Parameter & $\begin{array}{l}\text { Normal kidney function } \\
(n=12)\end{array}$ & $\begin{array}{l}\text { Severely impaired kidney } \\
\text { function }(n=8)\end{array}$ & $\begin{array}{l}\text { ESRD on HD/HDF treatment } \\
\text { period } 1^{\mathrm{a}}(n=8)\end{array}$ & $\begin{array}{l}\text { ESRD on } \mathrm{HD} / \mathrm{HDF} \\
\text { treatment period } 2^{\mathrm{b}} \\
(n=8)\end{array}$ \\
\hline \multicolumn{5}{|l|}{$t_{\max , \mathrm{EPO}}(\mathrm{h})$} \\
\hline Median (min, max) & $8.00(6.00,8.00)$ & $12.00(8.00,24.0)$ & $10.0(8.00,12.0)$ & $12.0(8.00,12.0)$ \\
\hline \multicolumn{5}{|l|}{$E_{\max }(\mathrm{mU} / \mathrm{mL})$} \\
\hline Mean (SD) & $161(128)$ & $316(307)$ & $253(201)$ & $386(224)$ \\
\hline Median (min, max) & $128(20.2,491)$ & $191(32.9,721)$ & $187(20.5,544)$ & $350(31.7,692)$ \\
\hline \multicolumn{5}{|l|}{$\mathrm{AUC}_{\mathrm{E}, \text { last }}(\mathrm{h} \cdot \mathrm{mU} / \mathrm{mL})$} \\
\hline Mean (SD) & $1580(1110)$ & $13,000(24,600)$ & $4210(3230)$ & $5940(3810)$ \\
\hline Median (min, $\max$ ) & $1390(378,410)$ & $2510(420,72,600)$ & $3430(416,9190)$ & $6050(479,11,100)$ \\
\hline
\end{tabular}

$A U C_{E, \text { last }}$ baseline-corrected area under the effect-time curve up to the last quantifiable sample, $E_{\max }$ baseline-corrected maximum observed effect; ESRD, end-stage renal disease, $H D$ hemodialysis, $H D F$ hemodiafiltration, $S D$ standard deviation, $t_{\max , E P O}$ baseline-corrected time of maximum erythropoietin effect

${ }^{\text {a }}$ Subjects received a single roxadustat $(100 \mathrm{mg})$ administration $2 \mathrm{~h}$ after completion of a dialysis session on day 1

${ }^{\mathrm{b}}$ Subjects received a single roxadustat $(100 \mathrm{mg})$ administration $2 \mathrm{~h}$ prior to the start of a dialysis session on day 1 


\section{Conclusion}

In conclusion, this study describes the pharmacokinetic and pharmacodynamic profiles of roxadustat and its circulating metabolites in subjects with normal kidney function, severely impaired kidney function, and ESRD on CAPD/ APD or HD/HDF, and demonstrates that the AUC of roxadustat and its metabolites was higher in subjects with severely impaired kidney function or ESRD on HD/HDF compared with those with normal kidney function, whereas $C_{\max }$ was not affected by kidney impairment. Roxadustat and its metabolites were not significantly cleared by HD/HDF.

Acknowledgements Medical writing/editorial support was provided by Rosalba Satta, PhD, and Elizabeth Hermans, PhD, from OPEN Health Medical Communications, Chicago, IL, USA and funded by the study sponsor.

\section{Declarations}

Funding Roxadustat is being developed by FibroGen, AstraZeneca, and Astellas. This study was funded by Astellas Pharma, Inc.

Conflict of interest V. Kerbusch reported personal fees from Astellas Pharma, Inc. during the conduct of the study and outside the submitted work. B. Barroso-Fernandez, M. den Adel, and R. Kaspera were employees of Astellas Pharma, Inc. during the conduct of the study. P. Galletti was contracted by Astellas Pharma, Inc. during the conduct of the study. D. Groenendaal-van de Meent is an employee of Astellas Pharma, Inc. G. Klein has nothing to disclose.

Ethical approval All procedures performed in studies involving human participants were in accordance with the ethical standards of the institutional and/or national research committee and with the 1964 Helsinki Declaration and its later amendments or comparable ethical standards. The study protocol (NCT02965040) was reviewed and approved by two Independent Ethics Committees (East of England -Cambridge East Research Ethics Committee and Technische Universität Múnchen, Fakultät Fû́r Medizin-Ethikkommission).

Informed consent All study participants signed a written informed consent.

Data sharing Researchers may request access to anonymized participant level data, trial level data and protocols from Astellas sponsored clinical trials at www.clinicalstudydatarequest.com. For the Astellas criteria on data sharing see: https://clinicalstudydatarequest.com/Study -Sponsors/Study-Sponsors-Astellas.aspx.

Author contributions DGM, MA, and VK contributed to the study conception and design. BBF, PG, GKK, and VK contributed to the acquisition of data. GKK contributed to the analysis and interpretation of the data. DGM, MA, RK, and VK participated in the analysis and interpretation of the data and in the drafting of this article. All authors provided critical revision of the article for important intellectual content.

Open Access This article is licensed under a Creative Commons Attribution-NonCommercial 4.0 International License, which permits any non-commercial use, sharing, adaptation, distribution and reproduction in any medium or format, as long as you give appropriate credit to the original author(s) and the source, provide a link to the Creative Commons licence, and indicate if changes were made. The images or other third party material in this article are included in the article's Creative Commons licence, unless indicated otherwise in a credit line to the material. If material is not included in the article's Creative Commons licence and your intended use is not permitted by statutory regulation or exceeds the permitted use, you will need to obtain permission directly from the copyright holder. To view a copy of this licence, visit http://creativecommons.org/licenses/by-nc/4.0/.

\section{References}

1. Babitt JL, Lin HY. Mechanisms of anemia in CKD. J Am Soc Nephrol. 2012;23(10):1631-4.

2. KDOQI, National Kidney Foundation. KDOQI clinical practice guidelines and clinical practice recommendations for anemia in chronic kidney disease. Am J Kidney Dis, 2006. 47(5 Suppl 3):S11-145.

3. Del Vecchio L, Locatelli F. An overview on safety issues related to erythropoiesis-stimulating agents for the treatment of anaemia in patients with chronic kidney disease. Expert Opin Drug Saf. 2016;15(8):1021-30.

4. Johnson DW, Pollock CA, Macdougall IC. Erythropoiesisstimulating agent hyporesponsiveness. Nephrology (Carlton). 2007;12(4):321-30.

5. Gupta N, Wish JB. Hypoxia-inducible factor prolyl hydroxylase inhibitors: a potential new treatment for anemia in patients with CKD. Am J Kidney Dis. 2017;69(6):815-26.

6. Akizawa $\mathrm{T}$, et al. Intermittent oral dosing of roxadustat in peritoneal dialysis chronic kidney disease patients with anemia: a randomized, phase 3, multicenter, open-label study. Ther Apher Dial. 2019;24(2):115-25.

7. Chen $\mathrm{N}$, et al. Roxadustat treatment for anemia in patients undergoing long-term dialysis. N Engl J Med. 2019;381(11):1011-22.

8. Chen $\mathrm{N}$, et al. Roxadustat for anemia in patients with kidney disease not receiving dialysis. N Engl J Med. 2019;381(11):1001-10.

9. Groenendaal-van de Meent D, et al. Effect of multiple doses of omeprazole on the pharmacokinetics, safety, and tolerability of roxadustat in healthy subjects. Eur J Drug Metab Pharmacokinet. 2018;43(6):685-92.

10. Ladda MA, Goralski KB. The effects of CKD on cytochrome P450-mediated drug metabolism. Adv Chronic Kidney Dis. 2016;23(2):67-75.

11. Liu B, et al. Metabolic enzyme system and transport pathways in chronic kidney diseases. Curr Drug Metab. 2018;19(7):568-76.

12. Matzke GR, et al. Drug dosing consideration in patients with acute and chronic kidney disease-a clinical update from Kidney Disease: improving Global Outcomes (KDIGO). Kidney Int. 2011;80(11):1122-37.

13. Akizawa $\mathrm{T}$ et al. Oral roxadustat three times weekly in ESAnaive and ESA-converted patients with anemia of chronic kidney disease on hemodialysis: results from two phase 3 studies. Ther Apher Dial. 2019. https://doi.org/10.1111/17449987.13468.

14. Chen $\mathrm{N}$, et al. Roxadustat treatment for anemia in patients undergoing long-term dialysis. N Engl J Med. 2019;381(11):1011-22.

15. Chen $\mathrm{N}$, et al. Roxadustat for anemia in patients with kidney disease not receiving dialysis. N Engl J Med. 2019;381(11):1001-10.

16. European Medicines Agency. Guideline on bioanalytical method validation. Committee for Medicinal Products for Human Use 
(CHMP). Effective February 1, 2012. [cited 2019 December 20]; http://www.ema.europa.eu/docs/en_GB/document_library/ Scientific_guideline/2011/08/WC500109686.pdf. Accessed 20 Dec 2019.

17. Nolin TD, Frye RF, Matzke GR. Hepatic drug metabolism and transport in patients with kidney disease. Am J Kidney Dis. 2003;42(5):906-25.

18. Rowland Yeo K, et al. Modeling and predicting drug pharmacokinetics in patients with renal impairment. Expert Rev Clin Pharmacol. 2011;4(2):261-74.
19. Akizawa T, et al. Phase 3, randomized, double-blind, activecomparator (Darbepoetin Alfa) study of oral roxadustat in CKD patients with anemia on hemodialysis in Japan. J Am Soc Nephrol. 2020;31(7):1628-39.

20. Locatelli $\mathrm{F}$, et al. Targeting hypoxia-inducible factors for the treatment of anemia in chronic kidney disease patients. Am J Nephrol. 2017;45(3):187-99. 